\title{
Application of big data in healthcare: examination of the military experience
}

\author{
David Berry ${ }^{1}$ (D) \\ Received: 20 October 2020 / Accepted: 23 November 2020 / Published online: 29 January 2021 \\ (c) The Author(s) 2021
}

\begin{abstract}
Healthcare is fully embracing the promise of Big Data for improving performance and efficiency. Such a paradigm shift, however, brings many unforeseen impacts both positive and negative. Healthcare has largely looked at business models for inspiration to guide model development and practical implementation of Big Data. Business models, however, are limited in their application to healthcare as the two represent a complicated system versus a complex system respectively. Healthcare must, therefore, look toward other examples of complex systems to better gauge the potential impacts of Big Data. Military systems have many similarities with healthcare with a wealth of systems research, as well as practical field experience, from which healthcare can draw. The experience of the United States Military with Big Data during the Vietnam War is a case study with striking parallels to issues described in modern healthcare literature. Core principles can be extracted from this analysis that will need to be considered as healthcare seeks to integrate Big Data into its active operations.
\end{abstract}

Keywords Big data $\cdot$ Hospitals $\cdot$ Electronic health records $\cdot$ Communication $\cdot$ Delivery of health care

\section{Introduction}

The current era of healthcare is characterized by the use of "Big Data" to help improve the precision of practices and maximize the efficiencies within its systems. Big data is characterized by high volume, often real-time data from multiple sources in both structured and unstructured formats. This term has also come to apply to the advanced analytic methods that are required to identify patterns and trends in these large data sets. In healthcare, these can take the form of predictive analytics for complication risk, utilization of user behaviour analytics for personalized medicine, and genomic analysis for therapeutic tailoring.

Often, the acquisition of this data is a passive by-product of healthcare digital systems used for day to day work. It is expected that the growth of data will proceed exponentially as time passes [1]. One of the primary goals of healthcare, therefore, is to develop the strategies and tactics that are needed to cope with and integrate this flood of plenty into practical operations within healthcare.

David Berry

BerryD@sah.on.ca

1 Department of Nephrology, Sault Area Hospital, Sault Ste. Marie, ON, Canada
The experience of business with big data has led healthcare toward attempting to adapt these models to its own system needs. Applying business models to healthcare, however, has limitations because of their inherent nature as "complicated" systems versus healthcare which is better described as a "complex" system. The study and adaptation of other complex systems, such as the military, may provide valuable additional insights for healthcare to implement big data in the most effective manner. Historical precedents derived from the military experience of implementing big data during active operations do exist and can be analysed for practical insights as healthcare moves forward on this path.

\section{Complicated versus complex systems}

Business models of production are often highly complicated but with known and predictable variables. Action A produces result B. Healthcare, in contrast, exhibits the traits of a complex system. Complex systems consist of multiple autonomous components that interact in multiple and variable ways such that the output is the result of the interactions between components and is not usually predictable by the properties of the components alone. Sometimes, changing 
the order of interaction between the same components is enough to produce opposite results. This differs significantly compared to a complicated system. If you put the tires on a car in a different order, it will still roll. If you apply therapies to a patient in a different order, you may get vastly different results. Simply put, the output from complicated systems depends on the nature of the components. Complex systems depend on the nature of the interactions between components for their output. This fundamental difference limits the broad applicability of business models and their value to the experience of healthcare. Peter Drucker, considered a founder of modern management, identified these issues in the statement "Health care is the most difficult, chaotic, and complex industry to manage today [and the hospital is] altogether the most complex human organization ever devised." [2]

\section{Parallels between healthcare and military systems}

Military models, in contrast to business, share many parallels with healthcare and provide a system closer to the nature of healthcare with extensive real-world experience of using big data. Healthcare and the military both function in multiple, chaotic, and dynamic environments that are only predictable to a degree and for limited time intervals due to a high number of variables, speed of change, and multiple relationships between components that influence outcomes. These elements generate large quantities of data that require rapid, if not real-time, analysis to best inform decisions and maintain coordination between different specialists over multiple theatres of operation.

Given healthcare is in the formative stages of utilizing big data, looking at early examples in the military experience is of interest. The Vietnam War is a case study of how military operations have parallels to healthcare with regards to the application of big data to active operations. This allows derivation of some practical principles which healthcare may utilize to accelerate its own development in this area.

\section{The vietnam war: a case study for the use of big data in operations}

The Vietnam War was set in the early days of the Information Age and is one of the first examples of the military using big data during active operations. The systems used and the approaches taken during this conflict have been documented and studied extensively, which makes it a particularly useful case study.

Vietnam was the first war to utilize extensive, extended, largely electronic pathways of data stretching from the front-lines back to the highest levels of authority. Ideally, the two elements of increased data generation and broader potential access to these data would have complemented each other with improved overall situational awareness and, ultimately, outcomes.

This did not occur for a variety of reasons. One element was that communications were usually carried out through "official" channels within a hierarchical model under processes established during World War II when the available technology was far more rudimentary. Another issue was that lateral, or informal, information flows between groups and individuals on the front-line, although increasingly necessary to coordinate efforts between different specialists and increasingly possible due to the proliferation of personal communication equipment, suffered due to the frequent rotations of the troops making up these groups. This created a third effect where no-one really knew anybody else on a personal level, reducing trust between actors. This reinforced reliance on centralized command and control structures via the official channels. A fourth effect was that these channels were used to document communications and provide heightened security on these communications.

What developed out of this centralized approach, however, was an environment and culture where front-line troops, although more proximal to the information both in time and space, were less willing to make operational or even tactical decisions. This deferral of authority was passed up the chain of command on a routine basis. As such, decisions were often delayed to a degree that the action taken was no longer relevant to the changing environment of the front-line troops [3]. The United States Air Force was estimated to require twenty-four hours to organize required resources for planned missions which, against a highly mobile opponent as faced in Vietnam, did not meet the practical needs of the troops in the field [4]. The limitations of such centralization of information were also readily apparent in the modern era with the Joint Special Operations Task Force in Iraq. As noted by General McChrystal "The practice of relaying decisions up and down the chain of command is premised on the assumption that the organization has time to do so, or more accurately, that the cost of the delay is less than the cost of the errors produced..." [5].

Relying on a centralized command and communication structure vastly increased the volume of reports and had the secondary effect of increasing the so-called "urgency" of reports. As people recognized that communications flagged as lower priority had scant chance of getting through, they would upgrade the priority level of their own reports. Everything, therefore, became "High-Priority", and because of security concerns, more messages were designated "Top Secret". With more messages of this nature, bottlenecks invariably formed in the flow of information and contributed to the inability to use the information in a timely fashion [3]. 
This continues to be an issue in the modern military. The practical application of hard won intelligence data was a significant challenge with the Joint Special Operation Task Force in Iraq where much of the potential information was not utilized in a timely manner to influence operations, in part because too much was happening too quickly on an ongoing basis and the data was considered to be the purview of the intelligence teams separate from the tactical operatives in the field [5].

Efforts to manage the flood of information in Vietnam and use it to coordinate operations led the United States military to hire economics specialists to draw on the experience of business with big data. This leant itself to the adoption of a cost-benefit analysis approach to the war. Missions were chosen and evaluated on defined metrics by high-level, remote statistical analysis, often generated from Washington, D.C [3].

The metrics chosen were intended to be meaningful surrogates to what constituted success in the "business" of war. The metrics being targeted, however, often did not associate with practical outcomes in the field, were difficult to acquire accurately in a meaningful time-frame, and could not be verified easily. An example was the use of "body count" which was an accounting of the number of enemy personnel killed. This was utilized as a metric for "success" with the reasoning that if the United States inflicted more casualties than they experienced they were winning. Aside from the practical difficulties of verification, the resultant potential for gaming the data, and given the human tendency to inflate or suppress reported values to meet expectations of their superiors, it failed to take into account the many other variables that applied to a military force having a dominating influence in their theatre of operations, which is the main objective for any system, healthcare included $[3,6]$. Thus, big data did not solve any problems and often created new ones, further degrading efficiency rather than enhancing it.

\section{Healthcare and big data}

Although the United States Military in the Vietnam War vastly outperformed their opponents based on evaluation of metrics, they still lost the practical elements of the war and ultimately withdrew. Many additional factors played roles, of course, but the outcome of the Vietnam War illustrates that merely having Big Data does not guarantee success. It also illustrates the inherent difficulties of applying principles of complicated systems into a complex system environment.

The elements that resulted in this outcome are all present in the current healthcare environment to a greater or lesser extent. There is a rapid proliferation of electronic communications allowing almost everyone to be reached at anytime, anywhere, by anyone. There is an increasing development of specialization at all levels of healthcare organizations for a wide variety of tasks with a corresponding increase in the required degree of coordination needed between these specialists which is not always being realized [7].

There is the same increasing tendency for reduced lateral communication between these groups as increasing task specialization occurs, often referred to as "siloing". This is despite the proliferation of personal communication technology (e.g. cellphones, tablet computers, and electronic medical records (EMR)) which should, in theory, have the opposite effect of enhancing lateral interactions [7, 8]. In one study at a "contemporary state-of-the-art" hospital in the United States, a survey of users identified the EMR as introducing barriers to communication between specialties, reduced confidence that notes were being shared appropriately, and that there was inhibition of effective and timely communication between practitioners [9]. This is of concern given the recognition that interdisciplinary teams with effective communication between all members are essential to providing effective and efficient patient-centred care in the modern healthcare environment and that ineffective communication contributes to patient harm [9-11].

Adding to this information burden is the advancing state of technology, which has allowed for the proliferation of sensory equipment. This adds an ever-increasing amount of potentially available data at any given moment. Healthcare in the United States in 2011 was estimated to produce approximately 150 exabytes $\left(150 \times 10^{18}\right.$ bytes $)$ of direct data [12]. To manage this plethora of data, there is an increasing tendency towards centralization of many operational elements. This is intended to manage the variety of information flows and to compensate for the loss of lateral communication pathways by creating official communication channels, often in the form of an EMR. Despite the increasing availability of data, however, studies by the Institute of Medicine found that deaths related to medication errors increased from $44000-98000$ in 1998 to $210000-400000$ in 2011 [13, 14]. Thus, the potential to reduce harm and enhance communications through the use of EMRs and its associated data collection and monitoring elements falls far behind their use as documentation platforms.

The reliance on EMRs for communication also reflects an increased sense of personal risk, a symptom of reduced trust in the system, as documentation often tends to be considered under the rubric of medico-legal risk mitigation in the event of an adverse outcome [10]. The centralized communication trend has, however, been found to erode interpersonal communication, limit access to information in a timely fashion, and delay patient care actions [9]. It has also been demonstrated to increase the risk of negative outcomes for patients [11]. This then, paradoxically, increases the potential of medico-legal risk exposure for all care providers and the institutions. 
Along with the widespread increase in potential access and centralization of data there is an opposite trend toward limitations on the access to this data due to the sensitive nature of this information and concerns about security. Despite our increasing reliance on information flows in healthcare, restrictions are being increased, often through government legislation, on who can access the data following a need-to-know type of philosophy. This severely limits the potential advantages of the Information Age in medicine and may blunt innovation [15].

This same issue was seen in Vietnam and continues to persist even into modern conflicts. After the Iraq War in 2003, the Joint Special Operations Task Force struggled in their ongoing conflict with several insurgent groups. This was despite deploying vast resources in all spheres that dwarfed their opponents. The structures and processes being used were not substantially different from those utilized in Vietnam, particularly with regard to intelligence gathering, analysis, and application, with the same resultant limitations. The Task Force was only able to capitalize on the wealth of data streams available after changing their structure and processes to include a more open information sharing philosophy using an interdisciplinary team culture. These changes led to increases in efficiency and outputs, being able to conduct over 300 missions per month rather than 18 with the previous structure [5]. In healthcare, the need for secure sharing of sensitive information across a broader platform is equally difficult. It becomes even more challenging as the multi-morbidity of the population increases, requiring more specialists to be involved in a larger number of cases. The increased number of caregivers involved will, in turn, generate more data with the need to share information more broadly.

To utilize the abundance of data and information, healthcare has increasingly turned towards business models. This has led to moves towards a cost-benefit, algorithmic, predictive approach with healthcare institutions often employing experts drawn from the business community to help manage healthcare operations. Metrics are chosen to reflect processes and outcomes that have been deemed important. They are often used as markers for outcomes without a clear understanding of what outcomes truly define success. Frequently there is little understanding of the variables that influence the metrics themselves or how metrics influence each other. This makes it difficult to implement meaningful action plans to influence the key variables. Healthcare, therefore, is at risk of - if not already repeating - the same errors seen in our case study of the Vietnam War.

\section{Integration of big data into operations}

The efforts of the military to develop models that more fully exploit the promise of Big Data have some practical principles that can be extracted to help healthcare improve its own implementation. A number of principles can be identified that contributed to the promise of big data not being realized in the Vietnam War nor, currently, within healthcare.

The first principle is that the proliferation of sources of data rapidly increases the volume of data acquired and may overwhelm analytic capabilities thereby limiting the practical utilization of this data. As recently as 2011, it was estimated that only $5 \%$ of the data generated by the United States Navy ever reached an analyst. It was also estimated that analysts spent $80 \%$ of their time looking for the data and only $20 \%$ of their time analyzing the data [1]. Similarly, in healthcare it has been identified that the use of EMRs may make it more difficult to find information and increases the time necessary to find it [9]. The impact of the increased volume of data and information flow needs to be considered as we integrate Big Data into systems so that we can maximize the benefit toward improving operations. How we practically use Big Data can be viewed in terms of what has been termed the "information pyramid", described as four layers designated data, information, knowledge, and wisdom/understanding [16]. As ordering and patterning of the data progresses through each layer, a system experiences similarly increasing levels of operational order and alignment. The corollary is that there is a corresponding reduction in the inherent tendency toward disorder through the provision of feedback between system elements to maintain alignment. Healthcare must clearly define, to the greatest possible extent, core competencies desired from the system to maximally exploit the potential of the information pyramid. By understanding the elements of operation essential to a system meeting its objectives the appropriate variables and metrics can be monitored to provide the necessary feedback to minimize friction and maximize coordination within the system [17].

This highlights the second principle - processes and technology need to be implemented to make the increased data streams accessible in a practical way so that key data are highlighted and readily available. This essentially can take the form of "official" channels" or unofficial "lateral" channels. EMRs, as has been discussed, have largely taken the role of official communication. EMRs, if relied upon for data transfer and communication, however, have been demonstrated to reduce in-person communication, increase the likelihood of incomplete or piece-meal information flow, and reduce shared problem-solving opportunities $[7,20]$. This is similar to the previously discussed limitations of relying on official pathways in the military experience.

Lateral, organically developed, pathways develop when information is considered urgent or the "official" technological route is felt to be insufficient to convey sufficient nuance or provide information in a timely fashion compared to the pace of events $[7-10,18,19]$. They often take the form of direct verbal communication. Lateral organic pathways are 
a potentially untapped route of communication to increase both bandwidth and practical information sharing. Technology can be utilized to increase the opportunity for accessing lateral streams of information flow but only where gaps functionally exist. In one study, the use of cell-phones to facilitate communication between neurologists and radiologists led to increased satisfaction by the neurologists from improved communication. This was less apparent with the radiologists involved who found the previous process of using the pager system met their needs and the cell-phones were not of added value [8]. Technology alone, therefore, will not help where current processes are efficiently fulfilling needs but must be part of an evaluation of what information is required to be available to whom and in what time frame $[8,9]$. Further evaluation of organic communication pathways can allow exploitation of these naturally occurring routes of communication and facilitate their integration into the overall "official" process. By acknowledging and integrating these pathways they can also be monitored to ensure the information is accurate and relevant to the appropriate processes and that opportunities are identified to utilize technology enhancing their utility. Without this ongoing evaluation of information flow outside of the official pathways, which will be used regardless of policy due to their perceived utility, coordination and alignment between members of healthcare teams of care plan priorities will be difficult to maintain [21]. Incorporating different routes of communication can expand the options available to healthcare teams and create opportunities for team problem solving to manage complex and multi-morbid patients by allowing for increased interaction between team members.

The third principle is closely tied to the first two - data is only useful if it can be accessed in a timely fashion. This principle is influenced by restrictions placed on access to the data based on security concerns. Certainly, sensitive data must be safeguarded and practices to minimize external leakage need to be developed and followed. If these efforts impair healthcare workers from having awareness of and/or access to information sources, however, substantial degradations in outcomes should be expected.

In healthcare, effective communication of information has been highlighted as an essential component of improved patient safety and outcomes. It also facilitates the necessary aspects of teamwork and coordination that are required to provide the best care for patients, particularly those with multi-morbid or complex conditions [11]. Gaps in information availability can lead to differing patient care priorities between team members and differing coordination around setting and achieving patient care goals [21]. Achieving optimal information availability without unduly compromising security will require ongoing assessments of risk-benefit balance and tolerance of practitioners and patients for the risks associated with both these elements. Implicit in this is the need to develop a culture of trust in other team members and confidence that the access to data is secure and appropriate. Intelligence services in the United States were made acutely aware of the consequences of fragmented Big Data with the terrorist attacks of $9 / 11$. Specific alterations in their approach included adopting methodologies to integrate different data types from diverse sources, automatically label data to allow for improved analysis and evaluation of patterns, control access to data at a granular level, and investment in employees specialized in data-science [22]. The use of encryption and cryptographic protocols has also been shown to allow sharing of data between different groups without affecting security or privacy [23]. Healthcare must incorporate strategies like these to not only ensure personal and sensitive data is safe from cyberattack, but that it is also easily accessible when needed so as not to compromise patient care. This is particularly important for multi-morbid patients who may have multiple care-providers distributed across many institutions with information in multiple databases.

\section{Conclusions}

Overall, big data overwhelms the tried and tested hierarchical models due to the volume and rapidity of data accumulation. The reflex reaction is to further strengthen these "official" pathways to manage information flows. This often works in the business environment as the interactions between components remain constant and predictable. It is merely of question of scaling.

In complex systems, however, the impact of big data is to disrupt the interactions between components. This can lead to a great deal of friction when the official process is at odds with the organic process (i.e. what works in the field).

By adopting a focus on the connections between, rather than the characteristics of, each component, increased confidence in those connections should allow for improved lateral transfer of information and better flows of information within a healthcare network. It should also improve the ability to prioritize information due to the stronger trust relationships that will develop, with resultant improvements in system outcomes and information security. This can only be realized when the old processes of the system are aligned with the new requirements of the system.

Although the wider availability of real-time data has the promise of creating more networked organizations, vastly expanding both capacity and capabilities, this requires investment in strengthening the interactions between team members to make this information both accessible and secure. This needs to be done through the construction of cultural models that focus on multiple points of interaction between team members, not just through designated official 
channels, and ensuring care providers are trained in these models. Technology can help create more points of contact but does not solve underlying pathologies in the system itself. Technology will only help broken processes break faster and more thoroughly.

Big Data is attractive because of the perception that a better informed system will reduce uncertainty and improve outcomes. More data, however, does not always lead to success and, as the Vietnam War example illustrates, can actually degrade outcomes. Further study of the military experience will be helpful to avoid similar pitfalls and accelerate development of healthcare systems in the current rapidly changing environment. If we do not learn from these lessons, we are short-changing ourselves and, more importantly our patients.

Acknowledgements The author would like to thank John Arquilla for many educational and insightful discussions, Amanda Caputo SAH librarian for her infinite help, Dr. Andrew Webb and Lt. Col. Lance Knox for editorial assistance, and the Sault Ste. Marie Academic Medical Association for financial support during this endeavour.

Funding Sault Ste. Marie Academic Medical Association (no grant number applied).

\section{Compliance with ethical standards}

Conflict of interest The author declares no conflicts of interest

Open Access This article is licensed under a Creative Commons Attribution 4.0 International License, which permits use, sharing, adaptation, distribution and reproduction in any medium or format, as long as you give appropriate credit to the original author(s) and the source, provide a link to the Creative Commons licence, and indicate if changes were made. The images or other third party material in this article are included in the article's Creative Commons licence, unless indicated otherwise in a credit line to the material. If material is not included in the article's Creative Commons licence and your intended use is not permitted by statutory regulation or exceeds the permitted use, you will need to obtain permission directly from the copyright holder. To view a copy of this licence, visit http://creativecommons.org/licenses/by/4.0/.

\section{References}

1. Porche III IR, Wilson B, Johnson E-E, Tierney S, Saltzman E. Data flood: helping the navy address the rising tide of sensor information. Office of the United States Navy. https://www.rand. org/pubs/research_reports/RR315.html (2014). Accessed 30 June 2018.

2. Denning S. The best of peter drucker. Forbes. 2014. https://www.forbe s.com/sites/stevedenning/2014/07/29/the-best-of-peter-drucker/ Accessed 30 June 2018.

3. Van Creveld M. Command in war. Cambridge. Massachusetts: Harvard University Press; 1985.

4. Van Creveld M. The transformation of war. New York, NY: The Free Press.

5. McChrystal GS, Collins T, Silverman D, Fussell C. Team of teams: new rules of engagement for a complex world. London: Portfolio Penguin; 2015.
6. Arquilla J, Roberts N. Design in warfare. Monterey: Naval Postgraduate School; 2017. p. 40-61.

7. Robinson FP, Gorman G, Slimmer LW, Yudkowsky R. Perceptions of effective and ineffective nurse-physician communication in hospitals. Nurs Forum. 2010;45(3):206-16. https://doi.org/10. 1111/j.1744-6198.2010.00182.x.

8. Naeger DM, Jafri NF, Webb EM, Avrin D, Qayyum A. Can hospital-based cellular phones improve on-call communication? J Am Coll Radiol. 2014;11(11):1090-2. https://doi. org/10.1016/j.jacr.2014.03.005.

9. Bardach SH, Real K, Bardach DR. Perspectives of healthcare practitioners: an exploration of interprofessional communication using electronic medical records. J Interprof Care. 2017;31(3):300 6. https://doi.org/10.1080/13561820.2016.1269312.

10. Zwarenstein M, Rice K, Gotlib-Conn L, et al. Disengaged: a qualitative study of communication and collaboration between physicians and other professions on general internal medicine wards. BMC Health Serv Res. 2013;13(1):1-9.

11. Baggs JG, Schmitt MH, Mushlin AI, Mitchell PH, Eldredge DH, Oakes D, Hutson AD. Association between nurse-physician collaboration and patient outcomes in three intensive care units. Crit Care Med. 1999;27(9):1991-8. https://doi.org/10.1097/ 00003246-199909000-00045.

12. Strome TL. Healthcare analytics for quality and performance improvement. Wiley; 2013. ISBN: 978-1-118-51969-1

13. Kohn LT, Corrigan JM, Donaldson MS eds. To err is human: building a safer health system, v.627; Washington, D.D.: National Academics Press; 2000.

14. James JT. A new evidence-based estimate of patient harms associated with hospital care. J Patient Saf. 2013;9(3):122.

15. Smaradottir B, Fensli R. A case study of the technology use and information flow at a hospital-driven telemedicine service. In: The practice of patient centered care: empowering and engaging patients in the digital era. Amsterdam, Netherlands: IOS Press; 2017. p. 58-62.

16. Lucky R. Silicon dreams: information, man, and machine. New York: St. Martin's; 1989.

17. Cooper, JR. Another view of the revolution in military affairs. In: In athena's camp: preparing for conflict in the information age. Eds. Arquilla JR, Ronfeldt D. RAND; 1997. ISBN: 0-8330-2514-7

18. Feldman NE, Sollfrey W, Katz S, Dudzinsky SJ. Writer-toReader Delays in Military Communications Systems. Project AIR FORCE - United States Air Force. https://www.rand.org/pubs/ reports/R2473.html (1979). Accessed 30 June 2018.

19. Gilardi S, Guglielmetti C, Pravettoni G. Interprofessional team dynamics and information flow management in emergency departments. J Adv Nurs. 2013;70(6):1299-309.

20. Taylor SP, Ledford R, Palmer V, Abel E. We need to talk: an observational study of the impact of electronic medical record implementation on hospital communication. BMJ Qual Saf. 2014;23(7):584-8.

21. Evanoff B, Potter P, Wolf L, Grayson D, Dunagan C, \& Boxerman S. Can we talk? Priorities for patient care differed among health care providers. In: Henriksen K, Battles JB, Marks ES, et al., editors. Advances in patient safety: from research to implementation (Volume 1: Research Findings). Rockville (MD): Agency for Healthcare Research and Quality (US); 2005.

22. Vigilante $\mathrm{K}$, et al. Big data and the intelligence communitylessons for health care. NEJM. 2019;380(20):1888-90.

23. Agrawal R, Johnson C. Securing electronic health records without impeding the flow of information. Int J Med Informatics. 2007;76:471-9. 